\title{
IDENTIFICAÇÃO E QUANTIFICAÇÃo DO CRISTAL VIOLETA EM AGUARDENTES DE MANDIOCA (TIQUIRA)
}

Geraldino da Silva Santos, Edmar Pereira Marques, Hildo Antônio dos Santos Silva e Cícero Wellington Brito Bezerra* Departamento de Química, Universidade Federal do Maranhão, Av. dos Portugueses, s/n, 65080-040 São Luís - MA Aldaléa Brandes Marques

Departamento de Tecnologia Química, Universidade Federal do Maranhão, Av. dos Portugueses, s/n, Campus do Bacanga, 65080-040 São Luís - MA

Recebido em 11/3/04; aceito em 5/11/04; publicado na web em 17/2/05

\begin{abstract}
IDENTIFICATION AND CHARACTERIZATION OF CRYSTAL VIOLET IN CASSAVA SPIRITS (TIQUIRA). Tiquira is a traditional homemade alcoholic distillate produced in the Maranhão State (Brazil), gotten from cassava (Manihot esculenta, Crantz.). It can be normally found on street markets. Due to the addition of tangerine leaves, the original tiquira has a bluish color. Samples of this beverage were acquired in the local trade and analyzed from the spectroanalytical point of view. The results indicated that these drinks had been adulterated by the addition of crystal violet, a potencialy hazardous compound. The identification and quantification of crystal violet in 10 spiked samples was accomplished by UV-Vis spectrophotometry through the standard addition method. In order to verify the efficiency of the proposed method, experiments on the quantification and recovery were carried out and the results indicated a content of crystal violet in the $10^{-6}$ to $10^{-7} \mathrm{~mol} \mathrm{~L}^{-1}$ range.
\end{abstract}

Keywords: crystal violet; tiquira; spectrophotometry UV-Vis.

\section{INTRODUÇÃO}

A tiquira, do Tupi: tykir = cair gota a gota ${ }^{1}$, é a bebida destilada obtida a partir da sacarificação e fermentação do mosto da mandioca (Manihot esculenta, Crantz.). Originária da cultura indígena, a partir do fermentado cauim (do Tupi: Cao $\tilde{y}=$ vinho qualquer) ${ }^{2}$, a tiquira continua sendo produzida e bastante apreciada, principalmente, nos estados do Maranhão e Piauí. Atualmente, o Maranhão parece ser o único estado brasileiro produtor desta bebida. O levantamento mais recente do IBGE (1996) ${ }^{3}$ indica uma produção em torno de 580 t/ano dessa aguardente, equivalente a 640 mil L e a uma movimentação financeira anual, aproximadamente, de R \$ 500.000,00.

Apesar da produção da tiquira ser modesta em relação à da aguardente de cana-de-açúcar (aproximadamente 2 bilhões de L/ano $)^{4}$, alguns fatores justificam o seu estudo, tais como ${ }^{5,6}$ : a sua larga aceitação regional (toda a produção encontra mercado); as condições inadequadas, em termos de higiene, em que alguns alambiques trabalham; o fato da flora microbiana utilizada nas etapas de sacarificação e fermentação ser colhida ao acaso, ou seja, diversas linhagens selvagens competindo no processo, com implicações importantes na composição e qualidade do destilado (proporção e natureza dos secundários), tempo e rendimento da produção; um teor médio elevado de íons cobre e a presença marcante de íons $\mathrm{CN}^{-}$, os quais são precursores do carbamato de etila.

Outro interesse que essa bebida desperta é com relação à elucidação dos compostos responsáveis pela sua coloração. É prática comum entre os produtores a adição de folhas de tangerina (Citrus reticulata Blanco) ao vinho, durante a etapa da destilação. Estas folhas conferem uma tonalidade levemente azulada ao destilado, como já demonstrado ${ }^{7,8}$. Entretanto, a exemplo de outras aguardentes, como a Caninha Azuladinha, algumas tiquiras chegam à mesa do consumidor com uma coloração violeta. Ao contrário da tiquira levemente azulada, a tiquira violeta não perde sua colora-

*e-mail: cicero@ufma.br ção com o tempo e apresenta no espectro eletrônico um processo em $585 \mathrm{~nm}$, o qual é dependente do $\mathrm{pH}$.

Neste trabalho, demonstra-se que o cristal violeta $(\mathrm{CV})$ (cloreto de hexametilpararosanilina, Figura 1), um corante do grupo dos trifenilmetanos, está sendo adicionado a esta bebida, na condição de corante alimentício. Esta substância, desde 1890, é de amplo uso na medicina e como corante em processos industriais ${ }^{9,10}$. É um agente anti-séptico indicado para infecções, particularmente as oriundas de bactérias gram-positivas ${ }^{11}$, e alguns outros patógenos como, por exemplo, a Cândida sp., e bastante comercializada em soluções aquosas nas concentrações de 1 a $2 \%$.<smiles>CN(C)c1ccc(C(=C2C=CC(=[N+](C)C)C=C2)c2ccc(N(C)C)cc2)cc1</smiles>

Figura 1. Fórmula estrutural do cristal violeta (cloreto de hexametilpararosanilina)

\section{PARTE EXPERIMENTAL}

\section{Reagentes e soluções}

Todos os reagentes utilizados foram de pureza analítica: etanol P.A (Merck), $\mathrm{NaOH}$ (Merck), $\mathrm{HCl}$ (Merck), $\mathrm{KCl}$ (Merck), Cristal Violeta (Merck), com exceção da solução comercial de violeta genciana (Farmax) e dos corantes alimentícios artificiais (Arcolor), adquiridos no comércio local.

A água utilizada no preparo das soluções e limpeza das vidrarias foi, unicamente, destilada. 
Todas soluções hidroalcoólicas (etanol:água) foram preparadas com a mesma graduação alcoólica determinada nas amostras de aguardentes.

Foi preparada uma solução padrão de CV, em meio hidroalcoólico, na concentração de $1,00 \times 10^{-3} \mathrm{~mol} \mathrm{~L}^{-1}$. A partir dessa solução, uma série de soluções trabalho foi preparada, na faixa de concentração $10^{-4}-10^{-6} \mathrm{~mol} \mathrm{~L}{ }^{-1}$.

\section{Amostras}

As amostras comerciais de tiquira, oriundas das localidades Barreirinhas (MA) e Santa Quitéria (MA), foram adquiridas no comércio local (Mercado Central e Reviver - São Luís/MA). Foram também adquiridas amostras diretamente dos produtores dessas respectivas localidades.

\section{Equipamentos e métodos}

As leituras espectrofotométricas foram realizadas em um espectrofotômetro UV-Vis, marca VARIAN, modelo Cary 50, em cubetas de quartzo com caminho óptico de $1 \mathrm{~cm}$.

Espectros eletrônicos das tiquiras e de soluções hidroalcoólicas de CV foram obtidos em pHs 0,$0 ; 1,3 ; 1,8,4,0$ e 12 . Esses valores foram escolhidos em função da faixa de viragem do indicador $(0,0-1,8)^{12}$. Os pHs foram obtidos através do emprego de tampões de Clark e Lubs $(\mathrm{HCl} / \mathrm{KCl}$ e $\mathrm{NaOH} / \mathrm{KCl})$, de acordo com a literatura ${ }^{13,14}$.

As determinações quantitativas foram realizadas pelo método da adição de padrões, a volume constante, conforme descrito a seguir: em cinco balões volumétricos $(10 \mathrm{~mL})$ contendo $9,0 \mathrm{~mL}$ da amostra foram adicionados, respectivamente, $1,0 \mathrm{~mL}$ da solução: hidroalcoólica (30\%); 1,0 mL das soluções trabalho contendo CV nas concentrações de $10^{-5}, 5 \times 10^{-5}, 10^{-6}$ e $10^{-7} \mathrm{~mol} \mathrm{~L}^{-1}$. As leituras foram realizadas em $585 \mathrm{~nm}$. Em todos os casos, os coeficientes de correlação ficaram muito próximos da unidade $(0,9998-0,9996)$. A Figura 2 ilustra uma das curvas analíticas obtidas. Todas as análises foram feitas em triplicatas.

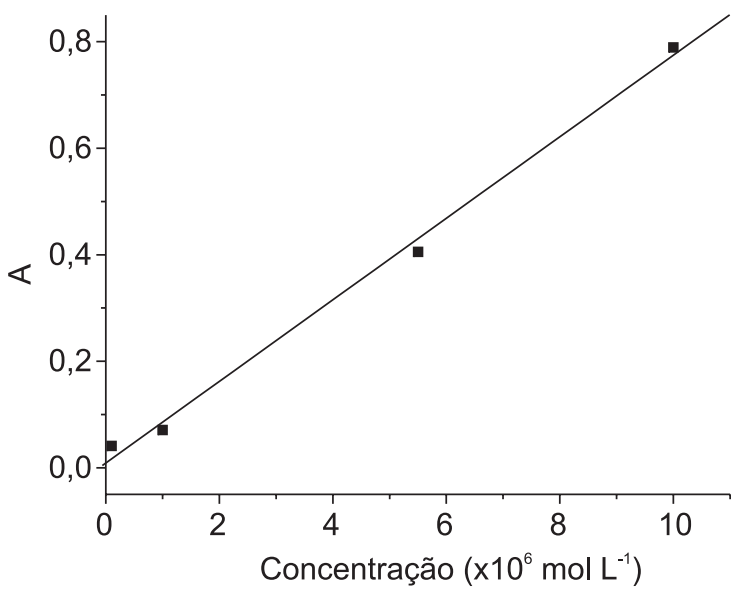

Figura 2. Exemplar das curvas analíticas construídas para análise do Cristal Violeta $(\mathrm{CV})$ em aguardentes de mandioca (tiquira). Método: adição de padrões. $A=0,10889+0,0091 C$. Cada ponto corresponde à média de três determinações

\section{RESULTADOS E DISCUSSÃO}

Os exemplares de tiquira encontrados no comércio de São Luís, rotulados como procedentes de Santa Quitéria e Barreirinhas, apre- sentaram uma coloração muito diferente dos coletados diretamente dos produtores, inclusive as dos correspondentes locais. Os espectros eletrônicos, Figura 3, mostram um processo em $356 \mathrm{~nm}$ para as tiquiras cuja coloração é proveniente dos compostos das folhas de tangerina e, um outro em $585 \mathrm{~nm}$, para as aguardentes de coloração violeta, encontradas no comércio local.

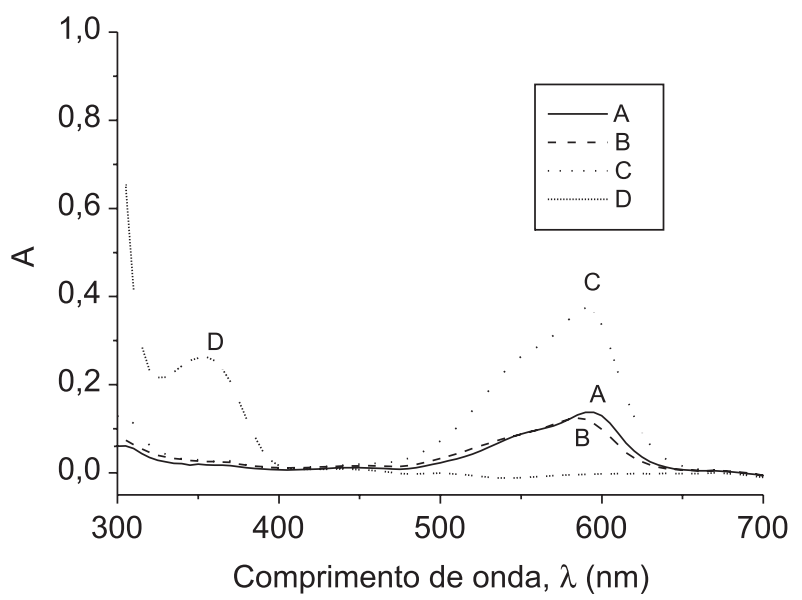

Figura 3. Espectro eletrônico da: A) solução de violeta genciana; B) aguardente de mandioca violeta; $C$ ) aguardente de mandioca violeta fortificada com $C V$ e D) aguardente de mandioca destilada em presença de folhas de tangerina

Alguns esforços foram feitos no sentido de se identificar a natureza do produto responsável pela coloração violeta dos destilados. Amostras de tiquiras incolores e azuladas foram contaminadas com anilina, anil imperial e corantes alimentícios azuis. Em nenhum dos casos houve coincidência de cor e de espectro eletrônico com os exemplares violetas. Entretanto, quando se experimentou uma solução de violeta genciana, amplamente utilizada pela população como anti-séptico, observou-se semelhança espectral. Também na Figura 3 estão representados os espectros eletrônicos de uma tiquira antes e após adição do CV, bem como de uma solução hidroalcoólica desta substância. Conforme pode ser observado, há um aumento na intensidade do processo em $585 \mathrm{~nm}$ quando se adiciona o CV a uma amostra de tiquira, sem a consequiente alteração no formato da banda.

Como o CV é um indicador ácido-base $(\mathrm{pKa}=1,0)^{15}$, foram verificadas também, em função do $\mathrm{pH}$, alterações na cor e nos espectros eletrônicos das tiquiras, e comparadas com as variações apresentadas por uma solução padrão de CV. Os pHs escolhidos foram 0,$0 ; 1,3 ; 1,8$ e 4,0, em função da zona de viragem do indicador. Os resultados mostram o mesmo padrão de comportamento para as duas amostras: com o aumento da concentração hidrogeniônica do meio, há um deslocamento hipocrômico da banda em $585 \mathrm{~nm}$, com a conseqüente alteração da cor (de violeta para verde). Esses experimentos foram realizados também com exemplares de tiquiras já violetas, mas fortificadas com CV. Os resultados foram os mesmos. Na Figura 4 estão ilustrados os espectros eletrônicos das tiquiras e dos padrões de violeta genciana, em função do $\mathrm{pH}$. A Figura 5 ilustra o espectro da tiquira e CV em pH 12, mostrando também a semelhança espectral na região do visível. Para esse $\mathrm{pH}$, foi verificado também um deslocamento hipsocrômico da banda de $356 \mathrm{~nm}$, em meio ácido, para, aproximadamente $290 \mathrm{~nm}$, em pH alcalino. Essa transição é observada apenas em tiquiras destiladas na presença de folhas de tangerina ${ }^{16}$.

Portanto, a simples acidulação do meio, por exemplo, adição de algumas gotas de ácido clorídrico, devido à alteração de cor 
pode ser uma metodologia viável para a verificação da presença do CV em destilados.

Os níveis de ocorrência do CV nas aguardentes de mandioca comercializadas em São Luís foram determinados por espectrofotometria UV-Vis, através do método da adição de padrões. A Tabela 1 resume os resultados obtidos. Foi encontrada uma variação na concentração de $\mathrm{CV}$ nas tiquiras na faixa de 7,765 x10 $10^{-7}-2,062$ $\mathrm{x} 10^{-6} \mathrm{~mol} \mathrm{~L}^{-1}$. A concentração média observada para as aguardentes analisadas foi de $1,236 \pm 0,568\left(10^{-6} \mathrm{~mol} \mathrm{~L}^{-1}\right)$.
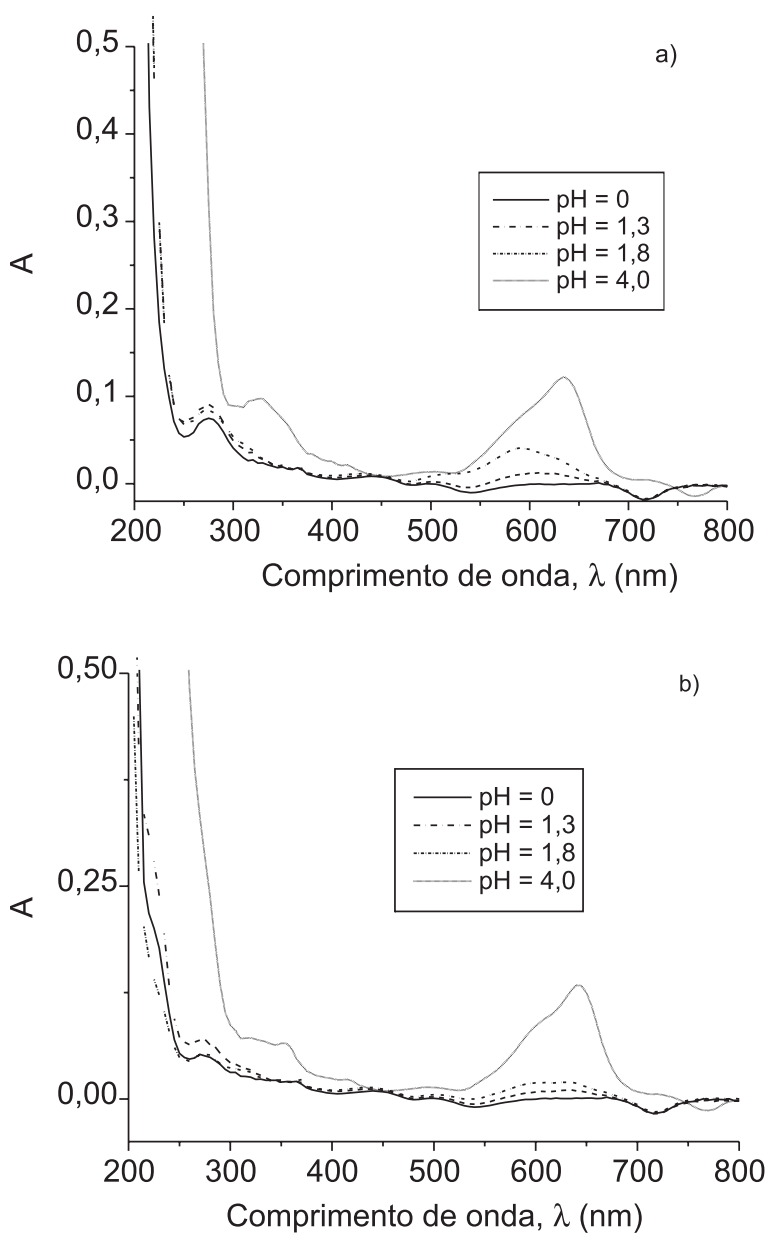

Figura 4.Espectros eletrônicos em diferentes pHs: a) aguardente de mandioca violeta e b) solução hidroalcoólica do $\mathrm{CV}$

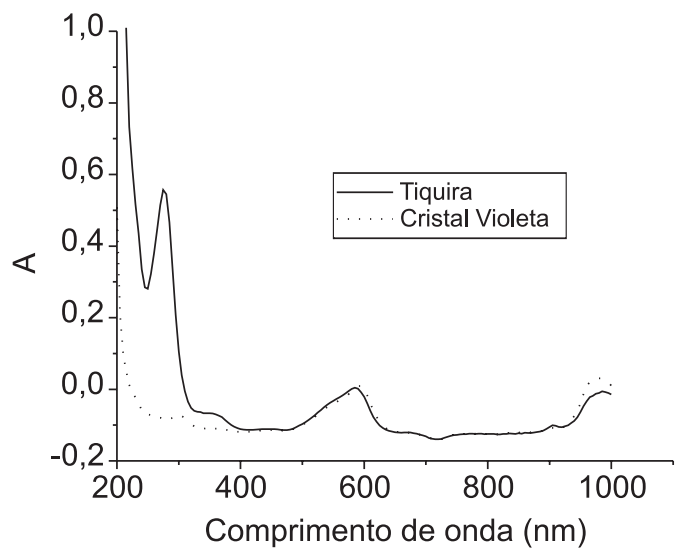

Figura 5. Espectros eletrônicos da tiquira e $\mathrm{CV}$ a $\mathrm{pH}=12$
Tabela 1. Níveis de ocorrência do $\mathrm{CV}\left(\mathrm{mol} \mathrm{L}^{-1}\right)$ nas aguardentes de mandioca $(n=3)$

\begin{tabular}{cc}
\hline Amostra & Concentração $\left(\mathrm{x} 10^{6} \mathrm{~mol} \mathrm{~L}^{-1}\right)$ \\
\hline $\mathrm{A}^{\mathrm{a}}$ & $0,776 \pm 0,001$ \\
$\mathrm{~B}^{\mathrm{a}}$ & $0,564 \pm 0,001$ \\
$\mathrm{C}^{\mathrm{a}}$ & $1,722 \pm 0,002$ \\
$\mathrm{D}^{\mathrm{b}}$ & $1,055 \pm 0,001$ \\
$\mathrm{E}^{\mathrm{b}}$ & $2,062 \pm 0,002$ \\
\hline Média & $1,236 \pm 0,568$ \\
\hline
\end{tabular}

$\mathrm{a}=$ Santa Quitéria - MA; b = Barreirinhas - MA

A literatura não registra casos de super doses, ou informações sobre a quantidade máxima tolerada por organismos com relação ao CV. Entretanto, ao consumo deste produto estão associados diversos efeitos colaterais ${ }^{11}$. Pode produzir, por exemplo, irritação e ulceração na membrana das mucosas, esofagites, laringites e traqueítes, náusea, vômitos, diarréia e dor abdominal. Além dessas sequielas, a literatura também classifica a violeta genciana como mutagênica e como poderoso veneno mitótico ${ }^{10}$.

Embora a metodologia proposta para a quantificação do CV em tiquiras tenha apresentado uma ótima linearidade, precisão e sensibilidade (variação na resposta do equipamento causado por um pequeno incremento na concentração do CV), os níveis de ocorrência encontrados justificam que se avalie também a eficiência da metodologia analítica proposta. Com essa finalidade, foram realizados estudos de recuperação ${ }^{17}$ na amostra real, e em um nível de fortificação: $0,10 \mathrm{mg} / \mathrm{mL}$. Na Tabela 2 estão apresentados os valores obtidos nos ensaios de recuperação. Todas as medidas foram feitas em triplicata. A percentagem média recuperada foi de 99,1 \pm $10,9 \%$, e os valores máximos e mínimos foram, respectivamente, $117,1 \pm 0,5 \%$ e $84,0 \pm 0,0 \%$, indicando a viabilidade e eficiência analítica do procedimento proposto.

Tabela 2. Estudos de recuperação do CV em matriz de tiquira

\begin{tabular}{cccc}
\hline Aguardente & $\begin{array}{c}\mathrm{m}_{\text {adi }} \\
(\mathrm{mg} / 100 \mathrm{~mL})\end{array}$ & $\begin{array}{c}\mathrm{m}_{\text {rec }} \\
(\mathrm{mg} / 100 \mathrm{~mL})\end{array}$ & $\begin{array}{c}\text { Recuperação } \\
(\%)\end{array}$ \\
\hline B & 10,1 & $9,75 \pm 0,05$ & $96,3 \pm 0,5$ \\
$\mathrm{~B}$ & 10,1 & $11,40 \pm 0,00$ & $112,6 \pm 0,0$ \\
$\mathrm{~B}$ & 10,1 & $8,50 \pm 0,00$ & $84,0 \pm 0,0$ \\
$\mathrm{~B}$ & 10,2 & $9,80 \pm 0,00$ & $95,9 \pm 0,0$ \\
$\mathrm{~B}$ & 10,9 & $10,60 \pm 0,00$ & $97,1 \pm 0,0$ \\
B & 10,3 & $9,35 \pm 0,05$ & $90,6 \pm 0,5$ \\
B & 10,1 & $11,85 \pm 0,05$ & $117,1 \pm 0,5$ \\
\hline Média & & & $99,1 \pm 10,9$ \\
\hline
\end{tabular}

\section{CONCLUSÃO}

A metodologia analítica proposta é conveniente para a determinação do $\mathrm{CV}$ em destilados. A acidulação dos mesmos, pela variação na cor, serve como indicativo da existência do CV no meio. Por ser um composto de intensa ação biológica, cujas conseqüências para o organismo ainda não estão bem esclarecidas, a prática de colorir a tiquira com o cristal violeta deve ser reprimida pelas autoridades competentes, através do devido esclarecimento da população.

\section{AGRADECIMENTOS}

Os autores agradecem ao Banco da Amazônia (BASA), CA- 
PES e CNPq pelo suporte financeiro e à Central Analítica/UFMA, pelo auxílio na realização de alguns experimentos.

\section{REFERÊNCIAS}

1. Cascudo, L. C.; Dicionário do Folclore Brasileiro, Ed. Itatiaia Ltda: Rio de Janeiro, 1993.

2. Cunha, A. G.; Dicionário da História das Palavras Portuguesas de Origem Tupi, Ed. Melhoramentos: Brasília, 1998.

3. Instituto Brasileiro de Geografia e Estatística - IBGE, Censo Agropecuário 1995-1996, Maranhão: IBGE, 1998, número 7.

4. Nascimento, R. F.; Bezerra, C. W. B.; Furuya, S. M. B.; Schultz, M. S.; Polastro, L. R.; Lima Neto, B. S.; Franco, D. W.; J. Food Comp. Analysis 1999, $12,17$.

5. Andrade Sobrinho, L. G.; Silva Filho, P. J.; Marques, E. P.; Bezerra, C. W. B.; Marques, A. B.; Lima Neto, B. S.; Franco, D. W.; Resumos do III Brazilian Meeting on Chemistry of Food and Beverages, São Carlos, Brasil, 2000.

6. Boscolo, M.; Tese de Doutorado, Instituto de Química de São Carlos, Universidade de São Paulo, Brasil, 2001.
7. Santos, G. S.; Monografia, Universidade Federal do Maranhão, Brasil, 2001.

8. Santos, G. S.; Bezerra, C. W. B.; Silva, F. C.; Marques, E. P.; Marques, A. B.; Resumos do $5^{\circ}$ Encontro de Química de Alimentos, Porto, Portugal, 2001.

9. Balabanova, M.; Popova, L.; Tchipeva, R.; Clinics in Dermatology 2003 , 21,2 .

10. Saquib, M.; Muneer. M.; Dyes Pigm. 2003, 56, 37.

11. Dacampo, R.; Moreno, S. N.; Drug Metab. Rev. 1990, 22, 161.

12. The Merck Index; $20^{\text {th }}$ ed., Merck Research Division: New Jersey, 1996.

13. Meites, L.; Handbook of Analytical Chemistry, $1^{\text {th }}$ ed., McGraw-Hill: New York, 1963.

14. Kolthoff, I. M.; Sandel, E. B.; Meehan,E. J.; Bruckenstein, S.; Quantitative Chemical Analysis, The Macmillan Company: London, 1969.

15. http://www.ping.be/at_home/indic-vir.htm\#liens, acessada em Agosto 2003

16. Santos, G. S.; Dissertação de Mestrado, Universidade Federal do Maranhão, Brasil, 2004.

17. Magalhães, L. L. S.; Dissertação de Mestrado, Universidade Federal do Maranhão, Brasil, 2003. 